Neurosurg Focus 17 (6):E12, 2004

\title{
Spinal epidural abscess: appearance on magnetic resonance imaging as a guide to surgical management
}

\author{
Report of five cases
}

\author{
Jonathon F. Parkinson, M.B.B.S., and Lali H. S. Sekhon, M.B.B.S., Ph.D., F.R.A.C.S. \\ Department of Neurosurgery and Spinal Injuries Unit, Royal North Shore Hospital, St. Leonards, \\ Australia
}

\begin{abstract}
Spinal epidural abscess is associated with considerable rates of morbidity and mortality despite its infrequent occurrence. Advances in magnetic resonance (MR) imaging technology have allowed easier diagnosis of this potentially devastating condition. It is also possible to predict the intraoperative appearance of each case of spinal epidural abscess prior to the procedure, based on the MR findings. Surgical treatment of this condition usually involves extensive decompressive laminectomy, which predisposes patients to spinal instability and deformity. Recent advances in surgical approaches to spinal epidural abscess have included the institution of less invasive techniques to manage this condition, including saline washes of the epidural space through catheters introduced via limited laminotomy. The cases reported here illustrate the ability to predict the intraoperative findings in patients with spinal epidural abscess, and to adjust the surgical approach accordingly to minimize the extent of potentially destabilizing procedures without impinging on the effectiveness of treatment.
\end{abstract}

\section{KEY WORDS - spinal epidural abscess - spinal infection - spinal cord compression • surgery - magnetic resonance imaging - myelopathy}

Infection involving the epidural space is seldom encountered, although when present it is often devastating. Spontaneous spinal epidural abscess occurs in 0.2 to 1.3 per 10,000 hospital admissions and typically affects immunosuppressed patients. ${ }^{3,8,11,16}$ Treatment has traditionally involved surgical decompression with or without drainage of the abscess, followed by prolonged antimicrobial therapy. Stabilization of the spinal column is also required occasionally. ${ }^{1,4,711-13,16}$ If the abscess extends over many segments, or if it extends in a panspinal fashion, surgical treatment can involve multilevel laminectomy, which over many levels can be destabilizing. ${ }^{2,9,11,12,14,16}$

In this report we present five patients with spinal epidural abscess to demonstrate the ability to predict the intraoperative appearance of this condition by using MR imaging. This is an update of previously published cases, with additional cases presented in support of our hypothesis. ${ }^{15}$ In our previous paper, we suggested that the consistency of the inflammatory tissue can be predicted preoperatively, in some cases allowing limited laminotomies with saline lavage to be performed using a feeding catheter. Consequently, the surgical approach could be planned preoperatively, based on the MR findings. The two additional cases presented here demonstrate this even

Abbreviation used in this paper: $\mathrm{MR}=$ magnetic resonance. more clearly, and also confirm the need for the treating surgeon to examine the MR images closely to offer appropriate treatment.

\section{CASE REPORTS}

\section{Case 1}

History. This 48-year-old woman was admitted to a peripheral hospital with a 24-hour history of left low-back pain that radiated down her left leg. She was also noted to have a low-grade fever. Over the following 3 days she experienced pain in her lower cervical region, a spiking fever, and tetraparesis in the right arm initially and progressing to involve both upper and lower limbs bilaterally. Blood cultures obtained during this time grew Streptococcus milleri.

Examination. On transfer to our institution, the patient was noted to have an asymmetric tetraparesis, with the right side more severely affected than the left. There was mild tenderness over her lower cervical spine. Her temperature was mildly elevated and the white blood cell count was $17.4 \times 10^{\%} / \mathrm{L}$. Her preoperative MR images confirming a panspinal epidural abscess are shown in Fig. 1 . Notably, the abscess enhanced in a heterogeneous fashion, with lack of central enhancement of the phlegmonous tissue. 


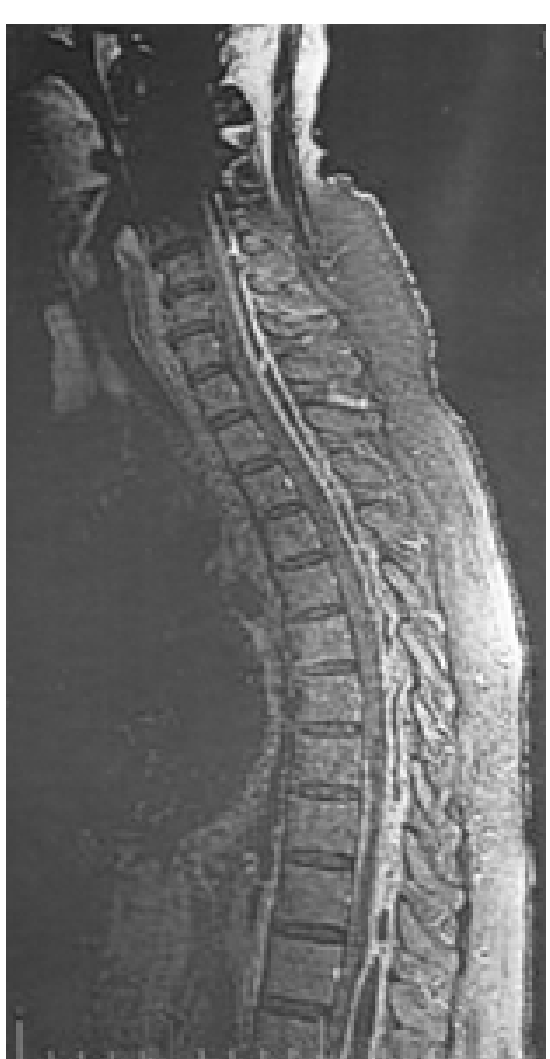

Fig. 1. Sagittal Gd-enhanced $\mathrm{T}_{1}$-weighted MR images demonstrating an extensive heterogeneous epidural collection. The majority of the collection is enhancing, representing phlegmonous tissue.

Operation. After the MR image was obtained, this patient was taken immediately to the operating room. After induction of general anesthesia, she was placed prone in a three-pin head fixation device and a midline incision was made from the occiput to the sacrum. Multilevel laminectomies were performed from C-2 to L-5, with the exception of T-6 and T-7. Frank pus was noted in the epidural space, and swabs were taken. These ultimately grew $S$. milleri, which was sensitive to penicillin. Notably, the consistency of the pus was such that there were portions that were liquid in nature, whereas other portions were solid and phlegmonous, more the consistency of granulation tissue. There were no intraoperative complications.

Postoperative Course. After surgery, intravenous benzyl penicillin was administered for a total of 6 weeks. Over this period there was a moderate improvement in the patient's neurological function. She was continued on a regimen of oral penicillin for a total period of 12 months of antibiotic treatment. Follow-up MR imaging studies demonstrated resolution of the epidural changes and no change in the sagittal alignment of the spinal column.

\section{Case 2}

History and Examination. This previously healthy 14year-old boy was admitted to our institution with a 3-day history of progressive weakness. When he was assessed in the emergency room his temperature was $37.9^{\circ} \mathrm{C}$. Neurologically, he had a flaccid quadriparesis with a Medi- cal Research Council score of 1-2/5 power in all motor groups. Anal tone was present but he required a urinary catheter. His initial white blood cell count was normal but his erythrocyte sedimentation rate was elevated at $90 \mathrm{~mm} /$ hour. He was taken immediately to the MR imaging unit, where images of his entire spine were obtained (Fig. 2). A panspinal epidural abscess was noted that extended from the craniocervical junction to the sacrum. Interestingly, the pattern of enhancement was not uniform, indicating that some portions were not granulation tissue, but rather were liquid in consistency.

Operation. The patient was taken to the operating room, general anesthesia was induced, and he was placed prone. Limited laminectomies were performed at C-3/4, C-7/T-1, T-5, T-12, L-3, and L-5/S-1. Liquid pus was encountered intraoperatively. The epidural space was washed thoroughly with saline solution delivered through an infant feeding catheter, and all purulent material was evacuated, decompressing the spinal cord. Subsequently, $S$. aureus was cultured from the wound and intravenous flucloxacillin was administered.

Postoperative Course. Over the next few weeks, the patient had an excellent neurological recovery. Repeated MR imaging showed resolution of the abscess, and after 6 weeks intravenous antibiotic therapy was discontinued. Six months after surgery, the patient was able to walk unassisted.

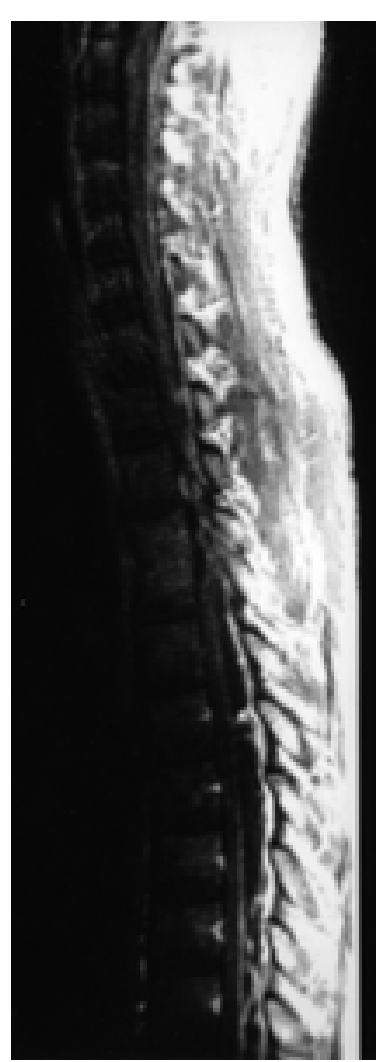

Fig. 2. Sagittal Gd-enhanced $T_{1}$-weighted $M R$ images again demonstrating an extensive epidural collection, although in this instance the majority of the collection is hypointense with an enhancing rim, representing liquid pus. 
Case 3

History. This 72-year-old man with type 2 diabetes was admitted to a peripheral hospital with a 5-day history of fever, lethargy, malaise, and hyperglycemia. He also noted a 2-day history of neck pain. Before his transfer he began to experience progressive weakness, initially in his left lower limb, but it spread to involve all four limbs.

Examination. On arrival at our institution, this patient had marked weakness in all four limbs, with the left side worse than the right. He had a preserved right triceps jerk reflex; however, all other limb reflexes were lost. He had a sensory level to pain and temperature of $\mathrm{C} 3$ on the left and T4 on the right. His admission MR image is shown in Fig. 3. Of note was the epidural enhancement from the occiput to the upper thoracic spine. In this case the enhancement was in parts uniform and in other parts patchy. The implication again was that the uniformly enhancing portions were liquid pus, with more solid phlegmon in the heterogeneous regions.

Operation. The patient was taken to the operating theater, general anesthesia was induced, and he was placed prone in a three-pin skull fixation device. The laminae from $\mathrm{C}-2$ to $\mathrm{T}-2$ were then exposed. Limited laminotomies were performed at C-7 and T-1, and liquid pus was found in the epidural space on incision of the ligamentum flavum (Fig. 4 left). The epidural space was the catheterized

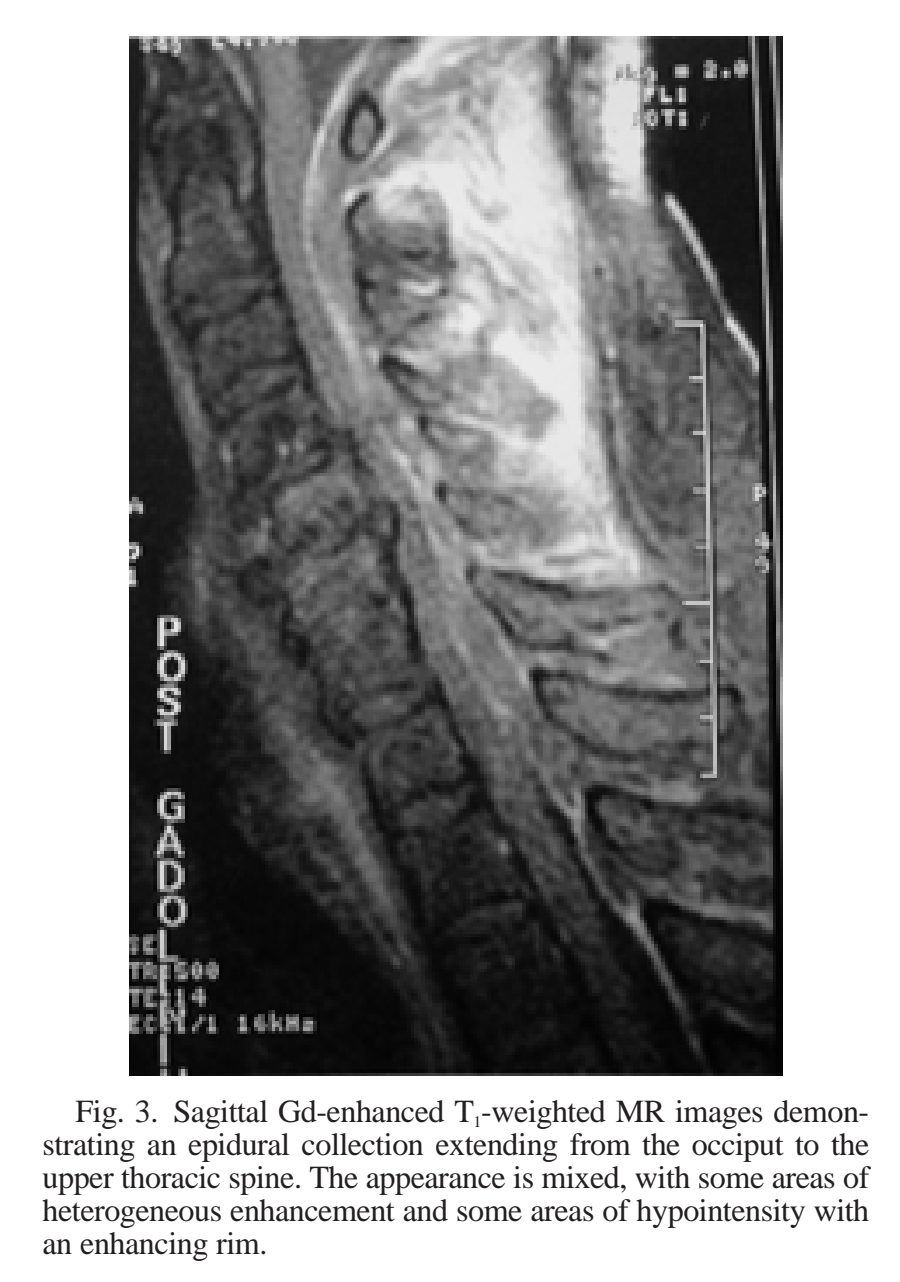

with a No. 8 French fine-bore infant feeding catheter (Mersk Indoplas Pty. Ltd., Sydney, NSW, Australia), and the space was then irrigated with copious amounts of normal saline until a clear return was obtained (Fig. 4 right). Laminectomies were then performed from C-3 to C-5.

Postoperative Course. Cultures of the epidural pus grew $S$. aureus, which was sensitive to penicillin, cephalexin, and cephazolin. The patient was treated initially with intravenous flucloxacillin, and this was soon changed to cephazolin. After a total of 6 weeks of intravenous therapy, his medication was changed to oral cephalexin. Serial MR images confirmed complete resolution of the epidural enhancement (Fig. 5).

\section{Case 4}

History. This 55-year-old man with a history of type 2 diabetes had a 4-week history of persistent neck pain and fluctuating arm symptoms. He initially sought chiropractic intervention and physiotherapy, but left arm and right leg weakness developed and he was referred to a local emergency room. Overnight his weakness progressed to a near-complete quadriparesis with minimal movement in both shoulders and no distal arm or leg movement.

Examination. The patient underwent emergency MR imaging, which revealed an anterior epidural mass causing spinal cord compression at the C4-5 vertebral body level (Fig. 6A). Gadolinium enhancement of the main mass was not present but the posterior longitudinal ligament enhanced, which was consistent with an anterior epidural abscess with associated osteomyelitis in the C-4 and C-5 vertebral bodies (Fig. 6B).

Operation. The patient was taken to the operating room and an emergency decompression was performed via an anterior approach. Thickened anterior longitudinal ligament was found. A vertebrectomy of the two levels was performed and liquid pus was encountered. A thorough debridement and decompression procedure was per-

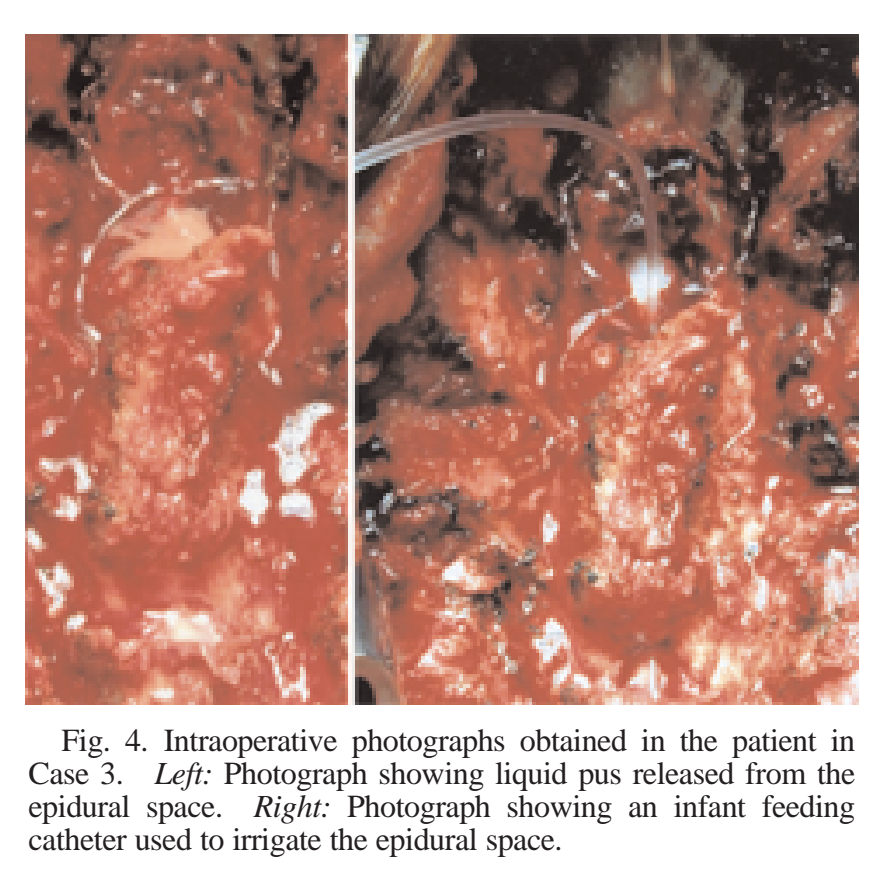




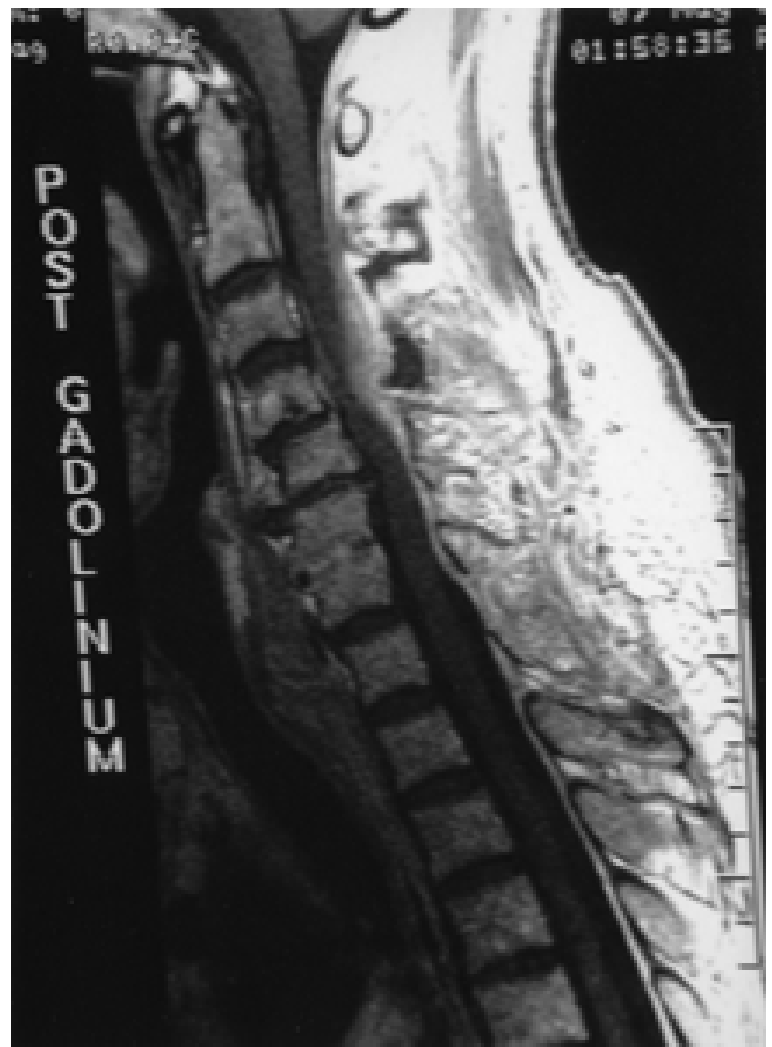

Fig. 5. Postoperative MR image obtained in Case 3 demonstrating effective decompression of the epidural abscess. A little residual dural enhancement is present.

formed, and a titanium mesh cage (Pyramesh; Medtronic Sofamor-Danek, Memphis, TN) filled with iliac crest autograft was placed in association with an anterior cervical locking plate (Atlantis; Medtronic Sofamor-Danek) (Fig. 7 left). During the same operating session a posterior approach was used to perform a C3-6 laminectomy and C2-7 instrumented fusion with polyaxial Vertex instrumentation (Medtronic Sofamor-Danek). Pars screws were inserted into the $\mathrm{C}-2$ level.

Postoperative Course. Postoperatively, the patient's inflammatory markers rapidly fell to normal. Cultures of intraoperative swabs yielded $S$. aureus, and the patient was maintained on intravenous and oral antibiotic therapy for 3 months. Despite his preoperative neurological state, he made a partial recovery of his quadriparesis with 3-4/5 strength in all limbs, and was able to feed himself and walk with aids at 6 months. His follow-up images at this time demonstrated complete cord decompression (Fig. 7 right).

\section{Case 5}

History and Examination. This 60-year-old man was admitted to our institution with a history of type 2 diabetes, 2 weeks of low-back pain, and a 1-week history of progressive leg weakness, constipation, and loss of bladder control. When he was assessed in the emergency room, he had 0-1/5 distal leg weakness and was unable to elevate his legs from the bed. Anal tone was reduced and

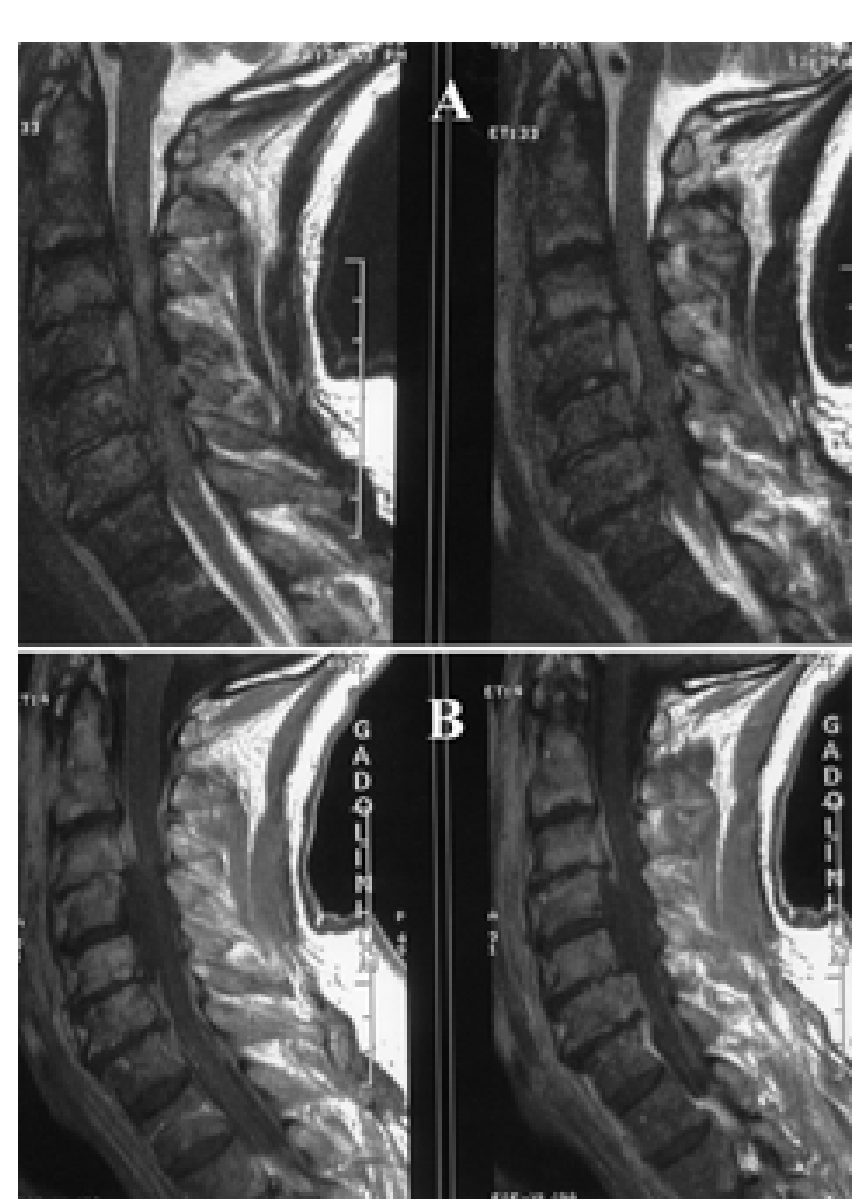

Fig. 6. Pre- (A) and postcontrast (B) sagittal $\mathrm{T}_{1}$-weighted MR images of the cervical spine obtained in the patient in Case 4 confirming ventral spinal cord compression at the C-3/4 level caused by a poorly enhancing ventral epidural abscess.

he had a high postvoid bladder residual volume. His inflammatory markers were raised and emergency MR imaging confirmed ventral compression of the cauda equina secondary to a mass at the L2-3 level. It was notable that on the MR image obtained with contrast material the central portion of this mass did not enhance, and the provisional diagnosis was liquid epidural abscess (Fig. 8).

Operation and Postoperative Course. The patient was taken to the operating room and exploratory surgery was performed. No pus was found but a wide posterior and lateral decompression was confirmed. Postoperatively, the patient continued to have elevated inflammatory markers with only a modest neurological improvement. Repeated MR imaging confirmed persistent compression and the site was again explored. As expected, liquid pus was found and drained. The collection was very ventral to the thecal sac. The patient has experienced slow improvement neurologically and in his inflammatory markers. At 3 months postsurgery he can ambulate independently but still has sphincteric disturbance.

\section{DISCUSSION}

Spinal epidural abscess, although rare, is associated with high rates of morbidity and mortality. ${ }^{1,4,7,13}$ The initi- 


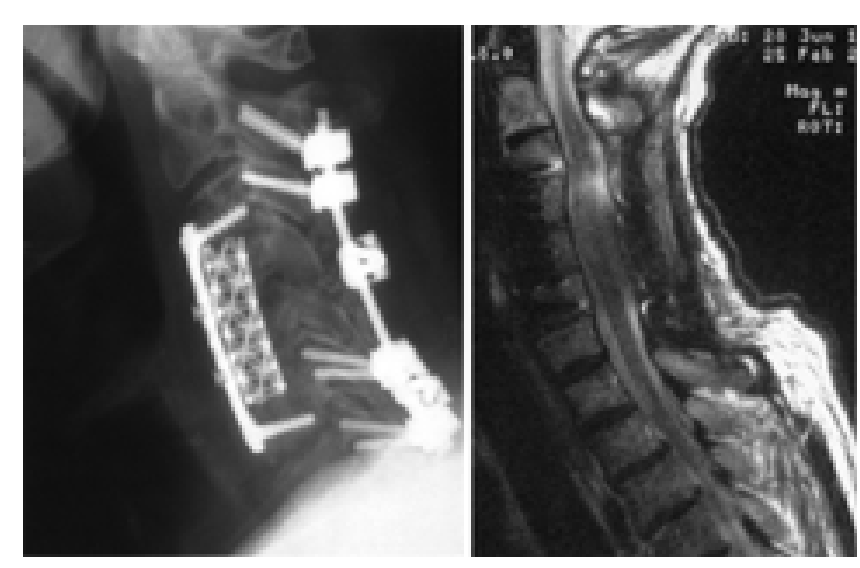

Fig. 7. Postoperative neuroimaging performed 6 months after surgical intervention. Left: Lateral cervical spine $\mathrm{x}$-ray film confirming placement of the instrumentation without development of hardware failure or kyphosis. Right: Postoperative $\mathrm{T}_{2}$-weighted sagittal MR image of the cervical spine demonstrating complete decompression of the spinal cord.

ation of treatment is often late because of difficulties in recognizing the condition. ${ }^{7,12,13}$ As detailed in various reports, ${ }^{1,12}$ Morgagni is frequently credited with recognizing and characterizing pyogenic infection in the epidural space in the 1760s. Nevertheless, surgical treatment was not successfully performed until the early twentieth century. The causative organism most frequently implicated in spinal epidural abscess is $S$. aureus. ${ }^{4,11,16}$

\section{Appearance on MR Imaging}

A spinal epidural abscess tends to have low or intermediate intensity on $\mathrm{T}_{1}$-weighted $\mathrm{MR}$ sequences and high

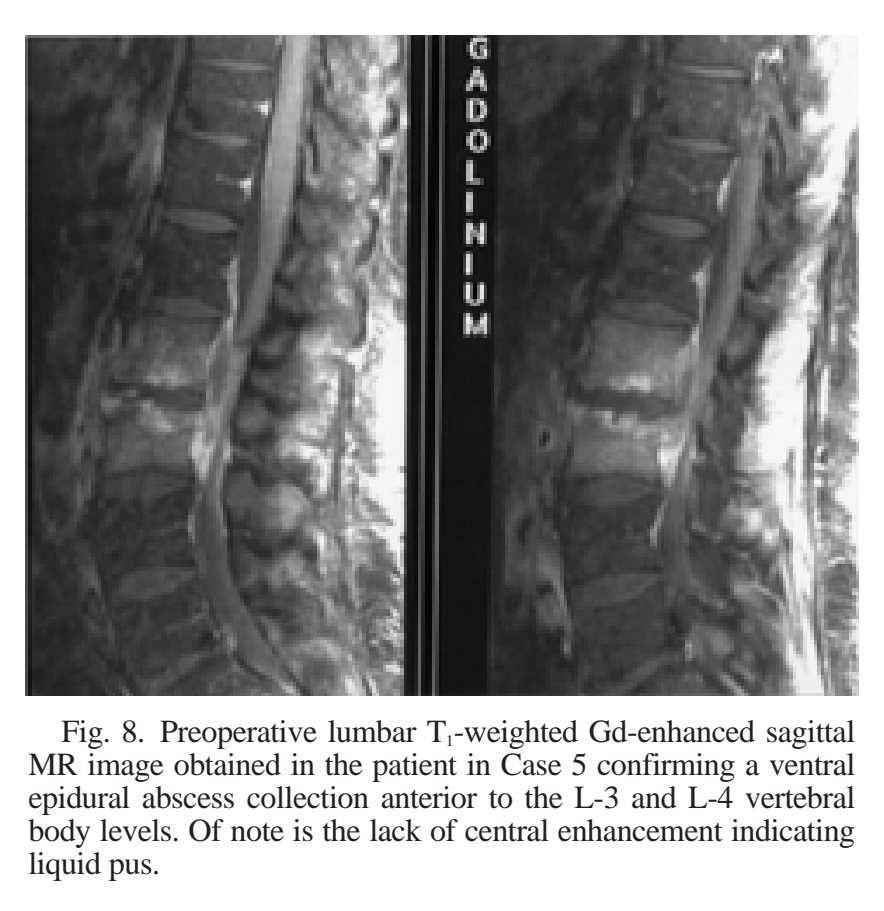

or intermediate intensity on $\mathrm{T}_{2}$-weighted images. The fluid portion of an abscess is usually markedly hyperintense on $\mathrm{T}_{2}$-weighted images, with corresponding hypointensity in $\mathrm{T}_{1}$-weighted MR images. ${ }^{8}$ Our cases have shown that Gd-enhanced MR images can aid in the definition of the age and consistency (that is, pus or granulation tissue) of the abscess. Liquid pus is associated with an area of low signal intensity on $\mathrm{T}_{1}$-weighted images, whereas a rim of tissue that enhances after the injection of Gd represents granulation tissue. ${ }^{8}$ Spinal epidural abscess is frequently associated with evidence of spinal cord compression on MR imaging. ${ }^{3}$

\section{Treatment Options}

When there is a neurological deficit associated with spinal epidural abscess, the treatment is usually decompressive surgery in conjunction with antibiotic therapy. ${ }^{1,4,7}$ 11-13,16 Surgical treatment has traditionally involved laminectomy with or without fusion. In instances in which anterior column elements are involved in the infectious process, destruction of bone and intervertebral disc may necessitate corpectomy and stabilization., ${ }^{4,11,16}$ More recently, minimally invasive techniques such as laparoscopic and thoracoscopic surgery as well as video-assisted open procedures have also been described. ${ }^{14}$ Intraoperative ultrasonography has also been used as an aid to assess the adequacy of decompression and the extent of drainage of the abscess. ${ }^{10}$ The insertion of catheters into the epidural space and abscess by using both open and percutaneous techniques has also been described. ${ }^{5,9}$

These techniques appear to be desirable because they offer a considerably less invasive treatment option for spinal epidural abscess. The difficulty has been in predicting which method of surgical treatment for spinal epidural abscess will be most effective and least invasive in each case. Although it was previously thought that rapid progression of the disease would be associated with purulent material, whereas a chronic infection would result in the accumulation of phlegmonous granulation tissue, a recent study has demonstrated that the intraoperative findings do not correlate with the rate of disease progression. ${ }^{11}$

In our first case, the MR findings led us to adopt a widespread decompressive approach, with extensive laminectomy. In contrast, the MR images obtained in the patient in Case 2 demonstrated extensive areas of liquid pus, so it was technically possible to treat the abscess with limited laminotomies and irrigation of the epidural space. The MR findings of a large area of liquid pus as well as areas more indicative of granulation tissue in our third case required a combination of the aforementioned techniques, and based on these MR findings we were able to plan our surgical approach. The postoperative MR findings demonstrated that this technique was effective in draining the abscess and adequately decompressing the spinal cord, while minimizing the risks of postlaminectomy kyphosis by using the somewhat minimally invasive technique of catheter irrigation through limited laminotomies. The extensive bone involvement in the fourth case, as appreciated best on the $\mathrm{T}_{2}$-weighted MR sequences, as well as the predominantly anterior location of the infected tissue, led to the use of an anterior surgical approach. Finally, the lack of liquid pus obtained during the first operation in the fifth 
case, contrary to expectations based on the MR appearance of the spine, resulted in inadequate decompression that necessitated a second procedure. Although no patient in this series suffered a postlaminectomy kyphosis, this is clearly a risk with such an extensive number of laminectomies, with the risk in some series reported to be as high as $21 \%, 2,2,6,9,12,14,16$

\section{CONCLUSIONS}

Spinal epidural abscess is a devastating condition if not diagnosed early and treated promptly and effectively. Preoperative MR imaging provides a noninvasive, accurate means of diagnosis in this condition. Recent advances in the surgical treatment of spinal epidural abscess have resulted in the increased use of less-invasive surgical techniques, thereby decreasing the morbidity associated with surgery in both the short and long term. In our series we demonstrate the ability to predict the intraoperative nature of spinal epidural abscess and thus treat the patient surgically in the least invasive but most effective manner.

\section{References}

1. Del Curling O Jr, Gower DJ, McWhorter JM: Changing concepts in spinal epidural abscess: a report of 29 cases. Neurosurgery 27:185-192, 1990

2. Fischer EG, Greene CS Jr, Winson KR: Spinal epidural abscess in children. Neurosurgery 9:257-260, 1981

3. Friedman DP, Hills JR: Cervical epidural spinal infection: MR imaging characteristics. AJR 163:699-704, 1994

4. Hlavin ML, Kaminski HJ, Ross JS, et al: Spinal epidural abscess: a ten-year perspective. Neurosurgery 27:177-184, 1990

5. Hori K, Kano T, Fukushige T, et al: Successful treatment of epidural abscess with a percutaneously introduced 4-French catheter for drainage. Anesth Analg 84:1384-1386, 1997

6. Kaptain GJ, Simmons NE, Replogle RE, et al: Incidence and outcome of kyphotic deformity following laminectomy for cervical spondylotic myelopathy. J Neurosurg Spine 93: 199-204, 2000

7. Khanna RK, Malik GM, Rock JP, et al: Spinal epidural abscess: evaluation of factors influencing outcome. Neurosurgery 39: 958-964, 1996

8. Lang IM, Hughes DG, Jenkins JP, et al: MR imaging appearances of cervical epidural abscess. Clin Radiol 50:466-471, 1995

9. Leonard J, Kaufman B: Treatment of holocord epidural abscess. Case illustration. J Neurosurg Spine 94:179, 2001

10. Mak KH, Au KK, Fung KY, et al: Spinal epidural abscess: a report of nine cases and the use of intraoperative ultrasonography. Aust N Z J Surg 66:287-290, 1996

11. Martin RJ, Yuan HA: Neurosurgical care of spinal epidural, subdural, and intramedullary abscesses and arachnoiditis. Orthop Clin North Am 27:125-136, 1996

12. McGee-Collett M, Johnston IH: Spinal epidural abscess: presentation and treatment. A report of 21 cases. Med J Aust 155: 14-17, 1991

13. Nussbaum ES, Rigamonti D, Standiford H, et al: Spinal epidural abscess: a report of 40 cases and review. Surg Neurol 38: 225-231, 1992

14. Parker LM, McAfee PC, Fedder IL, et al: Minimally invasive surgical techniques to treat spine infections. Orthop Clin North Am 27:183-199, 1996

15. Parkinson JF, Sekhon LH: Surgical management of spinal epidural abscess: selection of approach based on MRI appearance. J Clin Neurosci 11:130-133, 2004

16. Rea GL, McGregor JM, Miller CA, et al: Surgical treatment of the spontaneous spinal epidural abscess. Surg Neurol 37: 274-279, 1992

Manuscript received October 21, 2004.

Accepted in final form November 4, 2004.

Address reprint requests to: Lali Sekhon, M.B.B.S., Ph.D., F.R.A.C.S., Level 10, Tower B, 799 Pacific Highway, Chatswood, NSW 2067 Australia. email: surgeon@ spinalneurosurgery.com. 